\section{Author Correction: Codon-specific translation reprogramming promotes resistance to targeted therapy}

https://doi.org/10.1038/s41586-021-04074-1

Published online: 11 November 2021

Correction to: Nature https://doi.org/10.1038/s41586-018-0243-7

Published online 20 June 2018

Check for updates

Francesca Rapino, Sylvain Delaunay, Florian Rambow, Zhaoli Zhou, Lars Tharun, Pascal De Tullio, Olga Sin, Kateryna Shostak, Sebastian Schmitz, Jolanda Piepers, Bart Ghesquière, Latifa Karim, Benoit Charloteaux, Diane Jamart, Alexandra Florin,

Charles Lambert, Andrée Rorive, Guy Jerusalem, Eleonora Leucci, Michael Dewaele, Marc Vooijs, Sebastian A. Leidel, Michel Georges, Marianne Voz, Bernard Peers, Reinhard Büttner, Jean-Christophe Marine, Alain Chariot \& Pierre Close

In the version of this Letter initially published, not all the raw source data used to generate the graphs in the figures were presented at time of publication; they are hereby provided. Further, there are various corrections to the Figures and Extended Data Figures, as described below.
1. Some dots and error bars were unintentionally misplaced in the final formatting of some of the graphs, which could lead to data misinterpretation. Therefore, we provide corrected graphs for the following: Fig. 3f and Extended Data Figs. 1b, 1k, 2k, 2m, 4a, 6b, 8e and $9 \mathrm{~g}$. All stated statistics and conclusions remain correct.

2. In Extended Data Fig. 10i, the $y$-axis was wrongly labeled during the formatting of the figure. This has now been corrected.

3. In Fig. 4a, the Elp1 and Elp3 blots provided in the source data files did not correspond to those shown in the main figure. The correct source data files are now provided.

4. In Fig. 4c, the Elp3 and a-tubulin blots were provided in the source data file and wrongly referenced in the figure.

5. In Extended Data Fig. 3f, the original GLUT1 blot was provided in the source data file and wrongly referenced in the figure.

6. In Extended Data Fig. 9b, the ELP1 and ELP3 blots for SK-MEL-5 and A2058 lines were swapped in the main figure. The source date files were correct, and a corrected figure panel is here provided.

7. For Extended Data Figs. 1e, 4e, $8 \mathrm{a}$ and $8 \mathrm{~b}$, we correct mistakes in the labeling of the blots in the source data files.

8. For Fig. 3a source data files, we correct mistakes in the labeling in the legend of the table.

Finally, a question was raised to us regarding the specificity of the ELP1 signal in the RPL26 immunoprecipitation experiments shown in the Extended Data Fig. 10b. Therefore, to confirm that the ELP1 signal is specific in the anti-RPL26 immunoprecipitation, we reproduced the co-immunoprecipitation experiment using A2058 cells and the protocol provided in our manuscript. The antibody used in the manuscript was no longer commercially available, therefore in order to detect ELP1, we used the anti-ELP1 antibody (sc-100765; fully validated in our lab). This additional experiment shows that ELP1 binds RPL26 in A2058 cells, fully confirming and validating the results published in the manuscript in Extended Data Fig. 10b.

We thank the scientists who brought to our attention these unintentional inaccuracies. We are certain that the corrections and the raw source data now provided should fully clarify the conclusions published in the original manuscript.

Supplementary information is available in the online version of this Amendment.

(c) The Author(s), under exclusive licence to Springer Nature Limited 2021 